\title{
A Novel Coordinated Path Planning Method using k-degree Smoothing for Multi-UAVs
}

\author{
Liwei Huang, Hong Qu*, Peng Ji, Xintong Liu, Zhen Fan \\ School of Computer Science and Engineering, University of Electronic Science and Technology of China, Chengdu 610054, P. R. China
}

\begin{abstract}
Coordinated path planning for multiple Unmanned Aerial Vehicles (multi-UAVs) is a highly significant problem encountered in their coordinated control. In the interests of completing mission securely and efficiently, the advanced multi-UAVs control technology requires a universal smoothing method as well as a precise coordination strategy. In this paper, we propose a novel multi-UAVs coordinated path planning method based on the k-degree smoothing, a more complex environment consists of multiple threat sources of which is constructed. By employing the Improved Ant Colony Optimization algorithm, a k-degree smoothing method is also presented aiming at obtaining a more flyable path. Additionally, the multi-UAVs coordination algorithm is induced by k-degree smoothing, allowing the UAVs to arrive at the destination simultaneously or in an acceptable time interval. Finally, simulations of the comparison between the Improved Ant Colony Optimization and classic algorithm, the detailed smoothing method, and the coordination are respectively conducted to validate that the proposed approach is feasible and effective in multi-UAVs coordinated path planning problems.
\end{abstract}

Keywords: Multi-UAVs, Voronoi diagram, K-degree smoothing, Coordinated path planning

\section{Introduction}

Unmanned Aerial Vehicles (UAVs), the key to success for quantities of unmanned missions, have been widely applied in military and civilian fields, e.g., modern warfare, search and rescue under disaster circumstances, and weather monitoring, thanks to their low cost, strong viability and excellent maneuverability [1]. More than 70 percent of the UAVs are used for military purposes, e.g., battlefield reconnaissance and surveillance, positioning and fire, and damage assessment. They could also be employed for civilian applications, especially for border patrol, environment exploration, aerial photography, resource exploration, disaster monitoring and so on [2]. UAVs work in dangerous environments, and it is always important to keep their trajectories away from any type of threats and restricted areas. Therefore, the best routes are what minimize the risk of destruction of the UAVs, optimize some other planning criteria, and fulfill all the constraints which are imposed by the proposed mission, the physical characteristics of the UAVs, and the environment.

Seeking the optimal solution to trajectory planning for UAVs has been an active research area over the past few years. Roberge et al. [3] proposed Genetic Algorithm (GA) and Particle Swarm Optimization (PSO) algorithm to reduce the complexity of the path planning problem for UAV in a complex 3D environment. By appropriately formulating of a partially observable Markov decision processes (POMDPs) based algorithm action space, transition law, and objective function, UAV

\footnotetext{
${ }^{*}$ Corresponding author

Email: hongqu@uestc.edu.cn (Hong Qu)
}

could be guided for tracking multiple ground targets [4]. [5] formulated the global route planning problem for the unmanned aerial vehicles (UAVs) as a constrained optimization problem in the three-dimensional environment and proposed an improved constrained differential evolution (DE) algorithm to generate an optimal feasible route. Additionally, artificial bee colony algorithm combined with evolutionary programming [6], fuzzy quadtree framework [7], improved travelling salesman problem algorithm [8], and modified pulse-coupled neural network model [9] are also applied to the intelligent agent's path planning problems. There are other algorithms utilized to solve similar optimization problems, see [10], [11], [12], [13].

However, single agent is limited by its capacity and load, which makes it unable to complete complex tasks. Multiple agents coordination, taking a collaborative approach to achieve a mission, is possible to solve this issue. Gu et al. [14] proposed a new active persistent localization (APL) scheme for a moving target using a multiple-robot system, in order to obtain more accurate and robust localization results. In terms of the trajectory coordination of multiple mobile robots transportation problem, Michal et al. [15] revised the classical prioritized planning and made an asynchronous decentralized variant of both classical and revised prioritized planning, which can find solutions much faster in large multi-robot teams. A co-evolutionary improved genetic algorithm (CIGA) for global path planning of multiple mobile robots was addressed in [16], which employed a co-evolution mechanism together with an improved genetic algorithm (GA). P. B. Sujit et al. [17] presented a multi-UAVs coordinated path planning method by selecting a path from the feasible set of safe paths in coordination with other UAVs us- 
ing a dynamic leader approach to cover the entire unknown region. An integrated multiple autonomous underwater vehicle (AUV) dynamic task assignment and path planning algorithm was proposed for a 3-D underwater workspace with a variable ocean current in [18] by combing the improved self-organizing map (SOM) neural network and a novel velocity synthesis approach. To maximize the collected information, another algorithm was illustrated in [19] for multiple Unmanned Air Vehicles (UAVs) path planning in 3D environment, by introducing a novel evolutionary operator in Genetic Algorithm and the utilization of mTSP. Additionally, voronoi spatial partitioning is often utilized for multiple agents environment modeling when performing certain tasks as it can be constructed expediently, based on which the terminal eligible path is obtained, as described in [20], [21], [22].

In this paper, we propose a novel coordinated path planning algorithm for multi-UAVs under Voronoi environment. Different from traditional Voronoi based path planning issues focusing on single threat in the environment, we take into account multiple threats, e.g., radar threat, terrain threat, missile threat, and different treatments are adopted for corresponding threats to simulate the actual battlefield in our environment modeling. Given the flyable capacity, an Improved Ant Colony Optimization algorithm (IACO) the heuristic information function and pheromone update method of which are redefined is applied to multi-UAVs initial path planning, and to deal with the trajectory smoothing problem, we creatively introduce a k-degree smoothing method. Additionally, considering that the existed methods are inefficient in solving the coordination problem, we propose the multi-UAVs coordinated path planning algorithm, ensuring the smoothness of the trajectories and multi-UAVs to reach the targets simultaneously (strong coordination) or with an acceptable time interval (weak coordination) and can be effectively applied to real time cooperative tasks.

This paper is organized as follows. Section 2 is devoted to the environment modeling in terms of Voronoi diagram and the weights set of Voronoi edges. The detailed presentation of initial path obtained by the Improved Ant Colony Optimization algorithm, k-degree smoothing, and the coordination strategy are illustrated in Section 3. Several simulation experiments are conducted to confirm the high performance of the proposed algorithm in Section 4. Conclusions and outlooks are drawn in the last section.

\section{Environment Modeling}

Generally, previous works mainly considered about the length cost, as well as the threat of terrain factor and radar for the Voronoi diagram, and there is no difference in the treatment between terrain factor and radar cost, making the assessment of threat sources in the environment highly limited.

In this paper, we take into account not only the factors of length, terrain and radar, but also the threat of enemy missiles. Different treatments are employed for different types of threat sources to determine the cost of Voronoi edges. Visually, an il- lustration regarding multiple factors based on Voronoi diagram is displayed in Fig. 1.

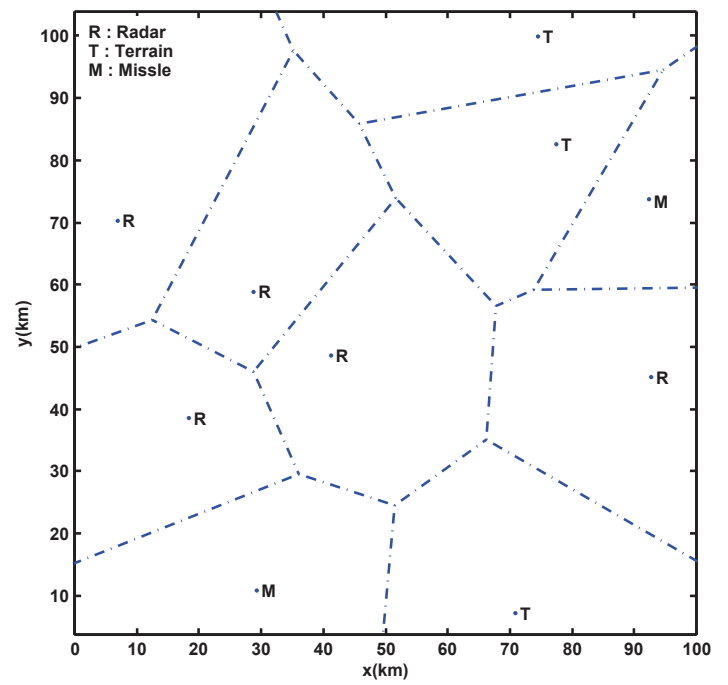

Figure 1: Environment modeling of the battlefield. R denotes the radar threat with detection ability, $\mathrm{T}$ denotes the constant terrain factor, $\mathrm{M}$ is the adversary missile with strike capability, the dotted line is generated by the Voronoi method.

In order to clearly illustrate multiple UAVs cooperatively path planning problem, some assumptions are given firstly:

(1) All the UAVs are located at different unique altitudes, hence there is no need for collision avoidance, and the path planning is simplified to operate in two-dimensional space;

(2) The UAVs fly with a constant rate, and the communication among them is reliable;

(3) The threat sources are treated as particles and generating vertices in Voronoi diagram.

As shown in Fig. 1, we denote the threat along edge $\underline{(i, j)}$ in the Voronoi diagram as

$$
J_{i j}=a \sum_{n=1}^{N_{r}} J_{i j, r}(n)+b \sum_{n=1}^{N_{t}} J_{i j, t}(n)+c \sum_{n=1}^{N_{m}} J_{i j, m}(n)+d J_{i j, l},
$$

where $a, b, c$, and $d$ are constant and fulfill that $a+b+$ $c+d=1 ; N_{r}, N_{t}$ and $N_{m}$ denote, respectively, the number of radars, terrain obstacles and guided missiles; $J_{i j, r}(n), J_{i j, t}(n)$, and $J_{i j, m}(n)$ are expressed as the $n-t h$ threat to edge $(i, j)$ from radars, terrain obstacles, and guided missiles, respectively; $J_{i j, l}$ represents the fuel cost of edge $(i, j)$, which can be defined as:

$J_{i j, r}(n)$-Radar threat: due to that the UAVs can be detected by the enemy radar, we take the radar transmit power as a threat level and denote the radar threat to UAVs by

$$
J_{i j, r}(n)=\int_{0}^{\frac{l_{i j}}{V}} \frac{Q_{n}}{R_{n}^{4}(t)} d t,
$$

where $V$ is the velocity of the UAV, $l_{i j}$ is the length of edge $(i, j), R_{n}(t)$ is the distance between the $\mathrm{UAV}$ and the $n-t h$ radar 
at time $t$, and $Q_{n}$ is the detecting power of $n-t h$ radar expressed as

$$
Q=\frac{P_{t} G A_{e} \sigma}{(4 \pi)^{2} R^{4}},
$$

where $P_{t}$ is power of the transmitter, $G$ is gain of the antenna, $A_{e}$ is the effective acreage of the antenna, $\sigma$ is section acreage of radar and $R$ is the distance from radar to object.

A computationally more efficient and acceptably accurate approximation to the exact solution is to calculate the threat cost at several locations along an edge: $\frac{1}{8} l_{i j}, \frac{3}{8} l_{i j}, \frac{5}{8} l_{i j}, \frac{7}{8} l_{i j}$. Thus, $\underline{J_{i j, r}(n)}$ can be expressed as

$$
\underline{J_{i j, r}(n)=l_{i j}\left(\frac{1}{d_{1 / 8, i j, n}^{4}+d_{3 / 8, i j, n}^{4}+d_{5 / 8, i j, n}^{4}+d_{7 / 8, i j, n}^{4}}\right) Q_{n},}
$$

where $d_{1 / 8, i j, n}$ is the distance from the $1 / 8$ point on the edge $(i, j)$ to the $n-t h$ radar threat.

$J_{i j, t}(n)$-Terrain threat: when penetrating in low-altitude, mountains and other complex terrain factors can be regarded as obstacles for UAVs, and the shorter the distance between the UAV and the terrain threat, the greater the threat of UAV. Thus, we define the influence of terrain threats as

$$
\underline{J_{i j, t}(n)=L_{n} e^{-k d_{i j}(n)}}
$$

where $L_{n}$ is the threat level of $n-t h$ terrain factor, $k$ is a coefficient and $\underline{d_{i j}(n)}$ is the Euclidean distance between the midpoint of edge $(i, j)$ and $n-t h$ terrain threat.

$J_{i j, m}(n)$-Missile threat: threat cost of the guided missile is decided by the hit rate and the attack power. Suppose $A$ is the penetration probability of the UAV, $B$ is the attack ability of the guided missile, and $\underline{p}_{i j}(n)$ is the detection probability of the $n-t h$ guided missile, the threat cost of guided missiles to edge $\underline{(i, j)}$ therein is defined as

$$
\underline{J_{i j, m}(n)=B(1-A) p_{i j}(n)} .
$$

$\underline{J_{i j, l} \text {-Fuel cost: }}$ since the fuel cost is proportional to the length of the edge, in our model, the fuel cost of edge $(i, j)$ is $J_{i j, l}$, defined as

$$
\underline{J_{i j, l}=\lambda l_{i j}}
$$

where $\lambda$ is the proportional coefficient and $\underline{l_{i j}}$ is the length of the edge $(i, j)$.

Consequently, after substituting $J_{i j, r}(n), J_{i j, t}(n), J_{i j, m}(n)$ and $J_{i j, l}$ in Eq. (1) with Eq. (4), Eq. (5), Eq. (6) and Eq. (7), the total threat of edge $(\overline{i, j)}$ can be expressed as

$$
\begin{aligned}
J_{i j}= & a \sum_{n=1}^{N_{r}}\left[l_{i j}\left(\frac{1}{d_{1 / 8, i j, n}^{4}+d_{3 / 8, i j, n}^{4}+d_{5 / 8, i j, n}^{4}+d_{7 / 8, i j, n}^{4}}\right) Q_{n}\right] \\
& +b \sum_{n=1}^{N_{t}} L_{n} e^{-k d_{i j}(n)}+c \sum_{n=1}^{N_{m}} B(1-\alpha) p_{i j}(n)+d \lambda l_{i j} .
\end{aligned}
$$

\section{Coordinated Path Planning Algorithm using k-degree S- moothing}

In this section, we propose a novel coordinated path planning method using k-degree smoothing for multi-UAVs. The framework of the trajectory planning for single UAV consists of three layers, as displayed in Fig. 2. The detailed initial path planning, smooth mechanism and coordination strategy are introduced in the next following subsections.

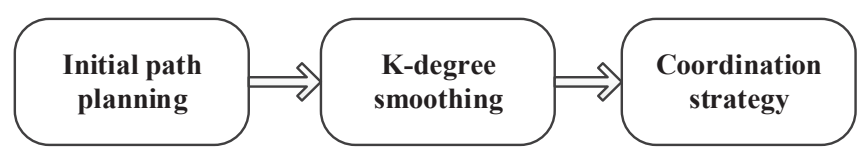

Figure 2: Trajectory planning framework for single UAV.

\subsection{Initial Path Planning for Multi-UAVs with Improved Ant Colony Optimization algorithm}

Ant Colony Optimization algorithm (ACO) was inspired by the observation on ant colony foraging behavior, and on that ants can often find the shortest path between food source and their nest. ACO has advantages of positive feedback of information, high parallelism, and optimization capability. Additionally, the foraging process of ant colony is quite similar with the path planning of UAV. Both of them are finding a best collision-free path from the start location to the destination.

To improve the efficiency of ACO, we introduce the heuristic information definition considering the Voronoi edge cost discussed in the last section as well as the pheromone update process to make it more feasible and efficient under the Voronoi environment. Taking the Voronoi edge cost into consideration of heuristic information makes UAVs find the optimal flight path with least threat cost, and renewing the pheromone of the global best path in fixed number of iterations, while the iteration best path in other iterations can accelerate the convergence speed. In this subsection, the Improved Ant Colony Optimization algorithm (IACO) is applied to solve the multi-UAVs initial path planning issue.

In IACO, ants select the next node according to the transition probability, that is

$$
P_{i j}^{k}(t)=\left\{\begin{aligned}
\frac{\left[\tau_{i j}(t)\right]^{\alpha}\left[\eta_{i j}(t)\right]^{\beta}}{\sum_{\gamma \in \text { allowed }}\left[\tau_{i \gamma}(t)\right]^{\alpha}\left[\eta_{i \gamma}(t)\right]^{\beta}}, & j \in \text { allowed }_{k} \\
0, & \text { otherwise }
\end{aligned}\right.
$$

where allowed $_{k}$ denotes the available nodes which ant $k$ can choose at node $i, \tau$ is the pheromone, $\eta$ is the heuristic information, $\alpha$ and $\beta$ are parameters that denote the impact of pheromone and heuristic information on transition probability, respectively.

For convenience notation, in IACO, we originally define the heuristic information as

$$
\eta_{i j}(t)=\frac{1}{J_{i j}(t) d_{j e}(t)},
$$


where $J_{i j}$ denotes the cost of edge $(i, j)$, which is calculated according to Eq. (8), and $d_{j e}$ represents the Euclidean distance from node $j$ to the target point $e$.

In one generation, once all ants complete the search, the pheromone of every edge is updated by

$$
\tau_{i j}(t+n)=(1-\rho) \tau_{i j}(t)+\Delta \tau_{i j}(t, t+n),
$$

where $\rho$ is the pheromone evaporation rate; $\Delta \tau_{i j}(t, t+n)$ is the pheromone increment laid on edge $(i, j)$ of the shortest path at the end of the cycle, which is be given by

$$
\Delta \tau_{i j}(t, t+n)=\sum_{k=1}^{m} \Delta \tau_{i j}^{k}(t, t+n)
$$

where $\Delta \tau_{i j}^{k}(t, t+n)$ denotes pheromone trail laid by the $k t h$ ant on the edge $(i, j)$ of the shortest path, and it is denoted as

$$
\Delta \tau_{i j}^{k}(t, t+n)=\left\{\begin{array}{cl}
\frac{Q}{L_{k}}, & \text { if ant } k \text { used }(i, j) \text { in its tour } \\
0, & \text { otherwise }
\end{array}\right.
$$

where $Q$ is a constant, and $L_{k}$ denotes the path length of ant $k$ in one generation.

To accelerate the convergence speed, a hybrid pheromone update method is employed. At fixed number of iterations, we update the pheromone on edges of the global best path. While in other iterations, we update only the iteration best path, as described in [23].

To further reduce IACO's probability of trapping into local optimal and improve the global search ability, pheromone is limited during the pheromone update phase of the shortest path at the end of one generation, which follows

$$
\tau_{i j}(t)= \begin{cases}\tau_{\min }, & \tau_{i j}(t) \leq \tau_{\min } \\ \tau_{i j}(t), & \tau_{\min }<\tau_{i j}(t) \leq \tau_{\max } \\ \tau_{\max }, & \tau_{\max }<\tau_{i j}(t)\end{cases}
$$

where $\tau_{\min }$ and $\tau_{\max }$ are the minimum and maximum pheromone trails on the edge.

The flowchart in Fig. 3 describes the detailed procedure of applying the Improved Ant Colony Optimization algorithm to a single UAV in the practical issue of multi-UAVs coordinated path planning.

The corresponding programming steps of IACO in solving multi-UAVs initial trajectory planning can be described as follows:

Step 1: Construct the Voronoi environment and calculate the cost of edges.

Step 2: Initialize the parameters of the algorithm, including $\alpha, \beta, \rho, Q, \tau_{\min }, \tau_{\max }$, as well as the number of ants $m$, the initial iteration number $N$ and the max iteration number $N_{\text {max }}$.

Step 3: Place the ants at the originating node, put the current node to the tabu table and choose the next node according to Eq. (9) until the target is reached.

Step 4: Choose the next ant and back to Step 3 until one iteration is completed.

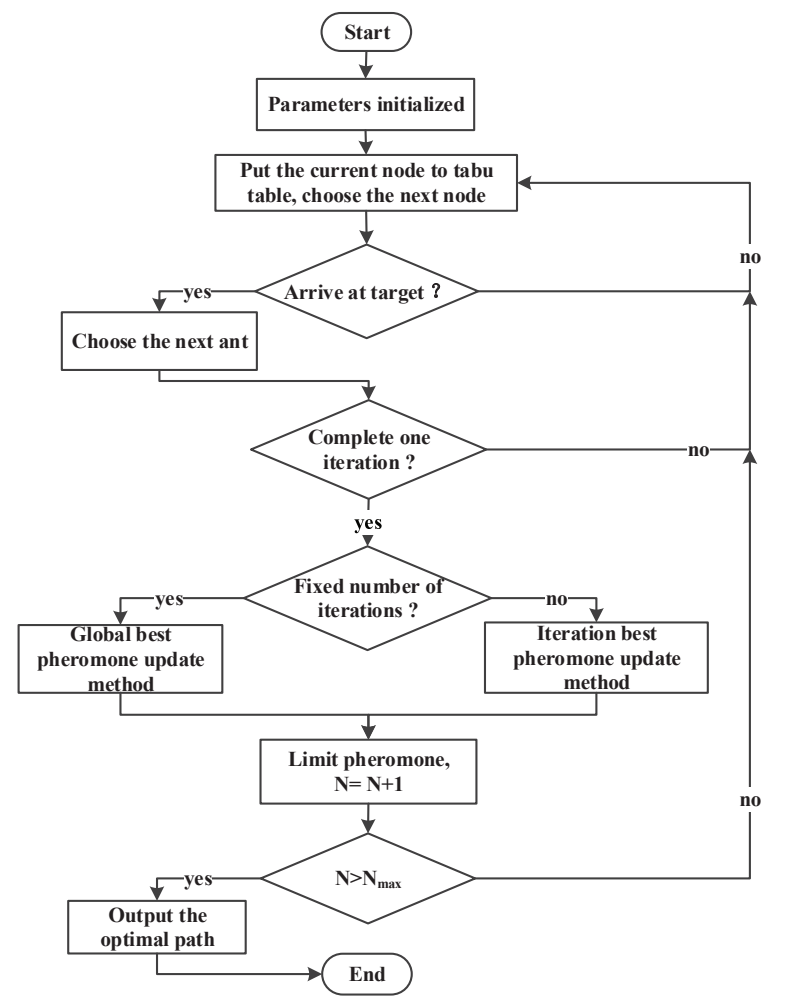

Figure 3: Flowchart of IACO applied to single UAV's path planning.

Step 5: Once fixed number of iterations is reached, update pheromone of the global best, otherwise update pheromone of the iteration best according to Eqs. (11) (13).

Step 6: Limit the pheromone based on Eq. (14), update the pheromone of edges and return to Step 3 until the terminating condition $N>N_{\text {max }}$ is satisfied, and output the optimal path.

\subsection{K-degree Path Smoothing}

From the Voronoi diagram modeled in the previous subsection, we can clearly find that there are some small angles which cannot meet the UAV's turn constraint, in other word$\mathrm{s}$ the searched initial path may not satisfy the actual demand. Therefore, we must smooth the path where two lines of Voronoi diagram connect with each other. In this subsection, we employ $k$-degree smoothing method to smooth the initial path, which provides the UAV with a series of dynamically feasible trajectories. And we prove that given the difference between the desired qualified final path length and the initial path length $\Delta l$, the corresponding smoothing degree $k$ can be computed through a certain equation.

\section{- Description of k-degree Smoothing Method}

Consider the waypoint path defined by three waypoints $w_{i-1}$, $w_{i}$, and $w_{i+1}$, shown in Fig. 4 . The unit vectors $\overrightarrow{q_{i+1}}, \overrightarrow{q_{i}}$ of corresponding path segments are denoted as

$$
\vec{q}_{i}=\frac{\overrightarrow{w_{i} w_{i-1}}}{\left\|\overrightarrow{w_{i} w_{i-1}}\right\|}
$$




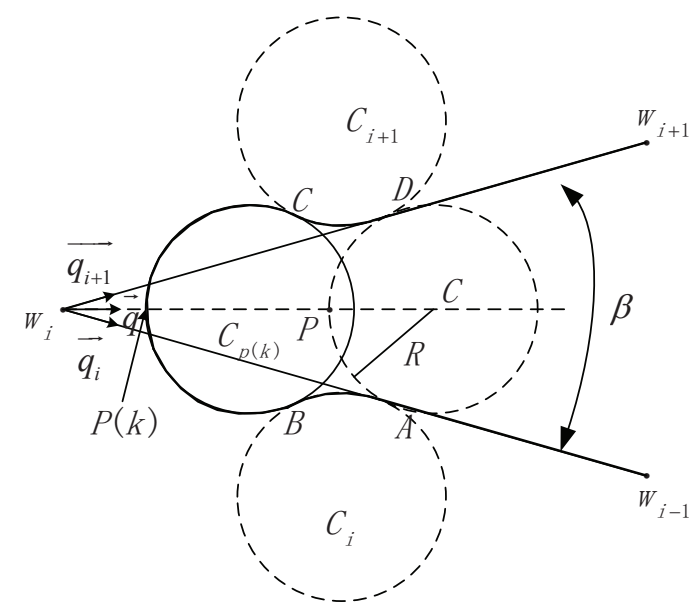

Figure 4: The dynamically feasible trajectories with smoothing degree k.

$$
\overrightarrow{q_{i+1}}=\frac{\overrightarrow{w_{i} w_{i+1}}}{\left\|\overrightarrow{w_{i} w_{i+1}}\right\|}
$$

Letting $\beta$ denote the angle between $\vec{q}_{i}$ and $\overrightarrow{q_{i+1}}$, and $\vec{q}$ the unit vector pointing along the bisector of $\beta$, we can get

$$
\cos \beta=\vec{q}_{i} \bullet \overrightarrow{q_{i+1}}
$$

and

$$
\vec{q}=\frac{\overrightarrow{q_{i}}+\overrightarrow{q_{i+1}}}{\left\|\overrightarrow{q_{i}}+\overrightarrow{q_{i+1}}\right\|} .
$$

Letting $C$ denote the inscribed circle with the minimum turning radius $R$, whose center lies on the bisector of the angle formed by the three waypoints, such that the circle intersect$\mathrm{s}$ both the lines $\overrightarrow{w_{i} w_{i-1}}$ and $\overrightarrow{w_{i} w_{i+1}}$ at exactly one point each. The bisector of $\beta$ can intersect the circle $C$ at two locations, and point $p$ is the intersection that is closest to $w_{i}$.

From Fig. 4, it can be seen that $\sin (\beta / 2)=R /\left(\left\|\overrightarrow{w_{i} p}\right\|+R\right)$. Through manipulation of this expression, the distance between $w_{i}$ and $p$ is found to be $\left\|\overrightarrow{w_{i} p}\right\|=R(1 / \sin (\beta / 2)-1)$. Suppose the inscribed circle $C$ moving towards to $w_{i}$ along the bisector of $\beta$, thus producing circle $C_{p(k)}$ and the $w_{i}$-closet intersection point $p(k)$.

Definition 1 Given the waypoint $w_{i}$ and point $p, k$ is the $s$ moothing degree following

$$
k=\frac{\left\|\overrightarrow{w_{i} p(k)}\right\|}{\left\|\overrightarrow{w_{i} p}\right\|} .
$$

Clearly, it can be found that $k \in[0,1], p(0)=w_{i}$, and $p(1)=P$.

As shown in Fig. 4, $C_{p(k)}$ is a circle of radius $R$, whose center lies in the bisector of $\beta$ and intersects $p(k)$. Additionally, letting $C_{i}$ be a circle of radius $R$ located outside angle $\beta$, and intersect segment $w_{i} w_{i-1}$ and circle $C_{p(k)}$ in exactly one location each. Define $C_{i+1}$ similarly.

A $k$-degree trajectory is constructed by the following sections, line segment $w_{i-1} A$, circular arcs $\widehat{A B}, \widehat{B p(k)}, \widehat{p(k) C}, \widehat{C D}$, and line segment $D w_{i+1}$, as shown in Fig. 4 . The $k$-degree trajectory is the unique and dynamically feasible trajectory that transitions from the waypoint segment $w_{i} w_{i-1}$ to the waypoint segment $w_{i} w_{i+1}$, passing directly through $p(k)$.

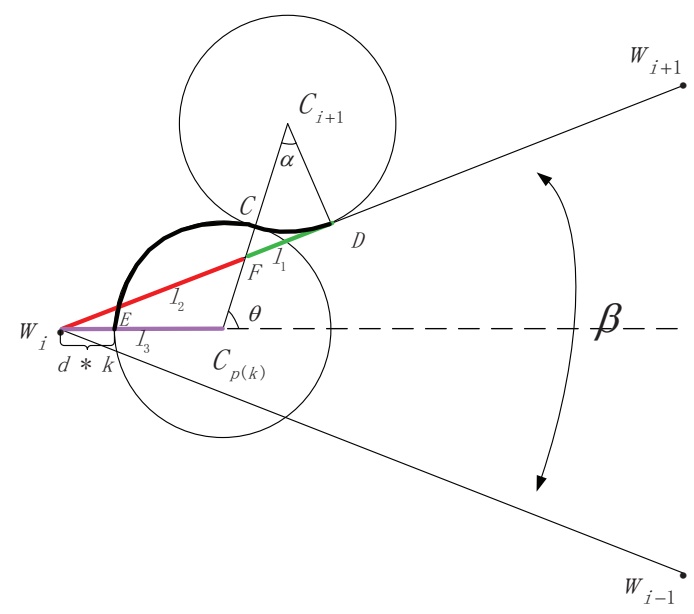

Figure 5: Smoothing principle using $k$-degree with angle $\beta$.

\section{- Theoretical Result}

Theorem 1 Given $\beta \in[0, \pi)$ and $k \in[0,1]$, the difference between the $k$-degree trajectory path length and the initial path length $\Delta l$ is expressed by the relevant smoothing degree $k$,

$$
\begin{aligned}
\Delta l= & 2\left[\left(2 \arccos \frac{2}{\sqrt{\tan ^{2} \alpha+1}}+\frac{\pi}{2}-\frac{\beta}{2}\right) R-R \tan \alpha-\right. \\
& \left.(k d+R) \cos \frac{\beta}{2}-(k d+R) \sin \frac{\beta}{2} \tan \alpha\right],
\end{aligned}
$$

where

$$
\cos \alpha=\frac{1}{2}\left[k\left(1-\sin \frac{\beta}{2}\right)+\sin \frac{\beta}{2}+1\right]
$$

Proof. As shown in Fig. 5, changes in length mainly involve arc $\widehat{E C}, \widehat{C D}$ and line segment $w_{i} D$. Clearly, we can get

$$
\theta=\frac{\beta}{2}+\frac{\pi}{2}-\alpha
$$

The total length of $\operatorname{arc} \widehat{E C}$ and $\operatorname{arc} \widehat{C D}$ is

$$
\widehat{l}=(\alpha+\pi-\theta) R .
$$

The length of line $F D$ is

$$
l_{1}=R \tan \alpha .
$$

The length of the line $w_{i} F$ is

$$
l_{2}=\frac{R(2 \cos \alpha-1) \sin \theta}{\sin \frac{\beta}{2} \cos \alpha} .
$$

Because of symmetry, we can get the difference $\Delta l$ expressed as

$$
\Delta l=2\left(\widehat{l}-l_{1}-l_{2}\right) .
$$


The distance between point $p$ and $w_{i}$ is

$$
d=\frac{R}{\sin \frac{\beta}{2}}-R .
$$

The distance between $w_{i}$ and the center of $C_{p(k)}$ is

$$
l_{3}=\frac{l_{2} \cos \alpha}{\sin \theta}=k d+R
$$

We can get the relationship of $k$ and $\Delta l$ expressed as

$$
\begin{aligned}
\Delta l= & 2\left[\left(2 \arccos \frac{2}{\sqrt{\tan ^{2} \alpha+1}}+\frac{\pi}{2}-\frac{\beta}{2}\right) R-R \tan \alpha-\right. \\
& \left.(k d+R) \cos \frac{\beta}{2}-(k d+R) \sin \frac{\beta}{2} \tan \alpha\right],
\end{aligned}
$$

where

$$
\cos \alpha=\frac{1}{2}\left[k\left(1-\sin \frac{\beta}{2}\right)+\sin \frac{\beta}{2}+1\right]
$$

which can be obtained by Eq. (25) and (26).

The results follow.

Specially, we demonstrate a situation of $\beta=\pi / 3$ and $R=10$, then the relationship of $\Delta l$ and the smoothing degree $k$ is obtained by the above equations and shown in Fig. 6. The figure shows that $\Delta l$ is an approximately monotone decreasing function of $k$ which follows Theorem 1 .

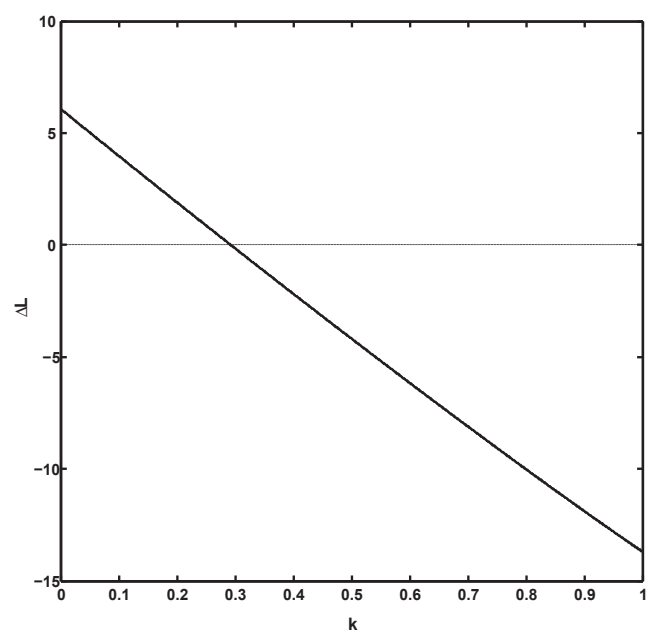

Figure 6: Relationship of $\Delta l$ and $k$ under the condition of $\beta=\pi / 3, R=10$.

From this theorem, we can conclude that when the circle $C_{p(k)}$ moves along the bisector of $\beta$, the shorter the distance between the center of $C_{p(k)}$ and $w_{i}$, the longer the total length of the path after smoothing is. As shown in Fig. 6, we can see that if $k=0$, then $\Delta l>0$ and if $k=1, \Delta l<0$. Since the function decreases monotonously, there is only one value of which can make $\Delta l=0$. That's to say, we can get the same path length as the waypoint path $w_{i-1} w_{i} w_{i+1}$ by means of changing the smoothing degree value of $k$.

\subsection{Multiple UAVs Coordination through Smoothing}

As described before, the smoothed UAV path using $k$-degree smoothing method can better describe the actual flight. However, the question arises of multiple UAVs coordination as well as their coordination degree. In this subsection, we present a novel multi-UAVs coordination strategy to solve the problems mentioned above.

That multi-UAVs starting from the initial location at the same time and arriving at the destination with minimal threat cost according to certain coordination standards, are considered as the goal of multiple UAVs coordinated path planning. Specifically, the cooperation standards in our paper mainly include:

(1) Strong coordination, which requires multi-UAVs reach the target simultaneously;

(2) Weak coordination, which denotes multi-UAVs reach the target in an acceptable time interval.

For one UAV, minimum time transition $(k=1)$, transitions through waypoints $(k=0)$, and transitions matching the coordination demand ( $k$ varies from 0 and 1 ), are the three fundamental ways of flight choices based on the $k$-degree smoothing approach. Based on single UAV's flight strategy, coordinations between multiple UAVs are described as follows.

Suppose that $n$ UAVs take part in the flying mission and each vehicle flies along its route at constant rate $V$. The length of trajectory planned by the ith vehicle is labelled as $L_{i}$, the upper bound and lower bound of the trajectory length after being smoothed for each UAV are denoted as $L_{i, \max }$ and $L_{i, \min }$ respectively, and ith UAV's path length is limited as

$$
L_{i} \in\left[L_{i, \min }, L_{i, \max }\right] .
$$

As for the UAVs formation with $n$ vehicles, suppose that

$$
A=\max \left\{L_{1, \min }, L_{2, \min }, \cdots, L_{n, \min }\right\},
$$

and

$$
B=\min \left\{L_{1, \max }, L_{2, \max }, \cdots, L_{n, \max }\right\},
$$

shown in Fig. 7.

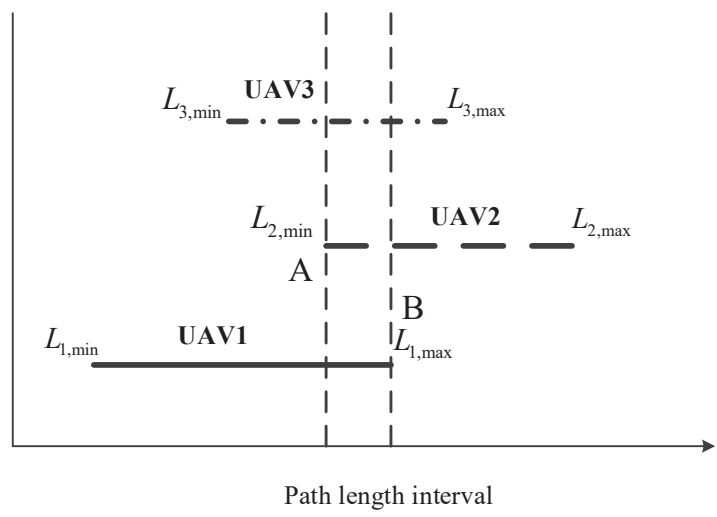

Figure 7: Feasible path length intervals of UAVs.

If $A-B \leq 0$, the multi-UAVs system can achieve strong coordination, which means that the trajectory interval of each UAV has a non-void intersection $I$, i.e.,

$$
I=\left[L_{1, \text { min }}, L_{1, \text { max }}\right] \cap\left[L_{2, \text { min }}, L_{2, \text { max }}\right] \cap \cdots \cap\left[L_{n, \text { min }}, L_{n, \text { max }}\right] .
$$


For the non-void intersection $I$, we assume such a length $L_{m} \in I$. The smoothed path of each vehicle must satisfy the path length $L_{m}$, thus $L_{m}$ can be regarded as the standard trajectory length in strong coordination circumstance. As for the special case $A-B=0$, there is only one element in the intersection $I$, and every UAV in the formation must take the unique choice as its standard trajectory length to reach the strong coordination goal.

Once the standard length is obtained, Greedy algorithm is applied to compute the $k$ value of every angle to determine the terminal trajectory. For the ith UAV's trajectory length $L_{i}$ with $p$ angles denoted as

$$
A n_{i}=\left\{\beta_{i 1}, \beta_{i 2}, \ldots \beta_{i p}\right\}
$$

According to the smooth principle, for the $j$ th $(j \in[1, p])$ angle of the ith UAV's initial path, there will be corresponding maximal increment and minimal decrement in the path length. Consider that $L_{m} \in I$, Eq. (27) is satisfied through smoothing manipulations on the angles, and there will also be no increment or decrement in some angles which can be achieved as we described in the former part.

Correspondingly, in weak coordination, multiple UAVs can reach the destination in a tolerable time interval. We assume the time interval is the minimal value of UAVs' tolerate time intervals $\Delta t_{i}$ expressed as

$$
\Delta t=\min \left\{\Delta t_{1}, \Delta t_{2}, \ldots \Delta t_{n}\right\}
$$

Since the velocities of vehicles are the same, the tolerate changes on path length will be $\Delta L(\Delta L=V \Delta t)$.

If $A-B>0$, intersection $I$ is empty, and the UAV formation cannot complete strong coordination. If $A-B \leq \Delta L$, weak coordination can be accomplished. Under this condition, each vehicle will have its own standard path length labeled as $L_{i m}$ $\left(L_{i m} \in[B, A]\right)$. Therefore, to reduce the computational cost, we denote the $L_{i m}$ as follows:

$$
L_{i m}= \begin{cases}L_{i m, \text { min }}, & L_{i m, \text { min }}, L_{i m, \text { max }} \in[B, A] \\ L_{i m, \text { min }}, & L_{i m, \text { min }} \in[B, A], L_{i m, \text { max }} \notin[B, A] \\ L_{i m, \text { max }}, & L_{i m, \text { min }} \notin[B, A], L_{i m, \text { max }} \in[B, A] \\ B, & L_{i m, \text { min }}, L_{i m, \text { max }} \notin[B, A]\end{cases}
$$

In the case of $A-B>\Delta L$, neither the strong coordination nor the weak coordination can be achieved.

In summary, when the UAVs coming from one formation determine their coordination strategy and the ultimate path length, each of them computes the corresponding path length differences respectively, and according to their own difference, they calculate the values of $k$ of angles in the path, as described in Thm. 1. Finally, the smoothed coordinated paths of each UAV are obtained. Through this approach, it is available for multiUAVs to meet the demand of arriving the destination simultaneously or in an acceptable time.

As stated above, the flowchart of multi-UAVs coordinated path planning is presented in Fig. 8.

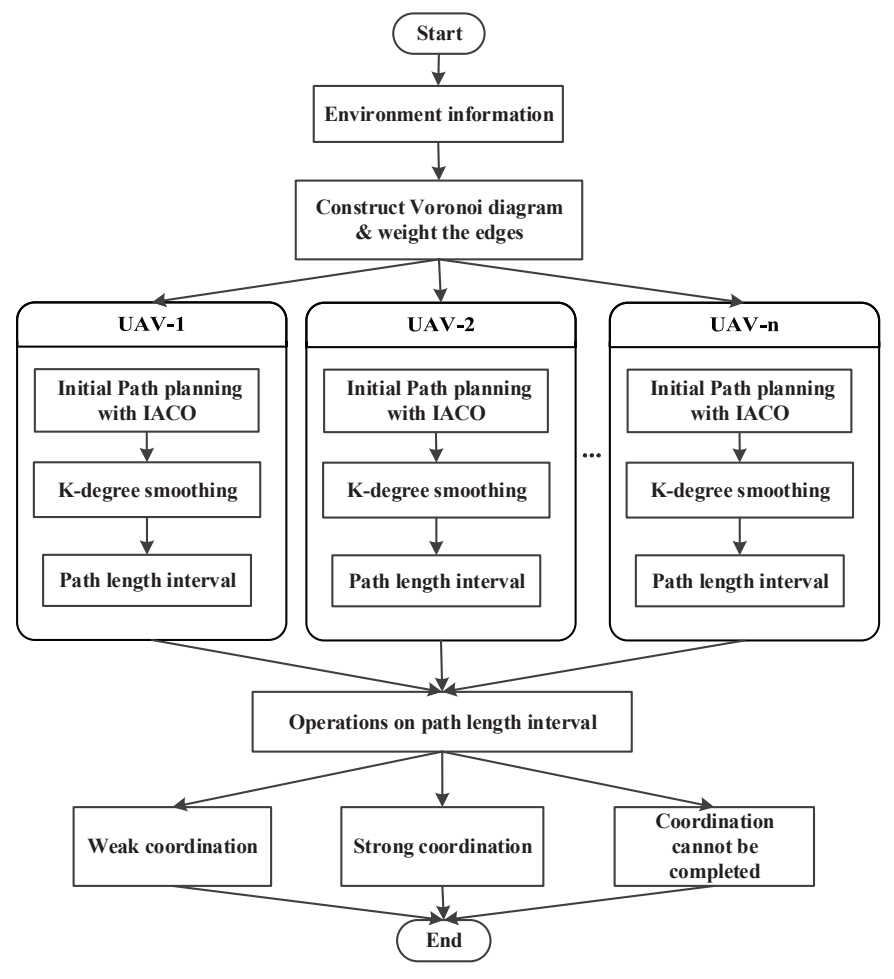

Figure 8: Flowchart of multi-UAVs coordinated path planning.

\section{Simulation and Analysis}

In order to demonstrate the feasibility and effectiveness of the proposed multi-UAVs coordinated path planning based on $k$-degree smoothing method, a series of simulations were conducted. These simulations were all implemented in Matlab with version 7.12 programming environment on an Intel Core 2 PC running windows 7 , and no ACO or multi-UAVs tools were introduced in the following simulations. In these simulations, the mission region $\left(100 \mathrm{~km}^{*} 100 \mathrm{~km}\right)$ is made up of known threat$\mathrm{s}$ generated randomly, as illustrated in Section 2. The shortest path is searched by the Improved Ant Colony Optimization algorithm, and $k$-degree smoothing method is applied to smooth the initial paths and achieve coordination for multi-UAVs.

\subsection{Convergence Rate Comparison of the Improved An- $t$ Colony Optimization algorithm and Ant Colony Opti- mization algorithm}

In the first simulation, performance comparison between the Improved Ant Colony Optimization algorithm (IACO) and Ant Colony Optimization algorithm (ACO) was conducted.

First, we initialize the parameters referred to subsection 3.1 listed as follows: the population size is 50, the number of maximal iterations is 150 , the pheromone weight $\alpha$ and heuristic information weight $\beta$ in Eq.(9) are 1 and 5 respectively, pheromone evaporation rate $\rho$ in Eq. (11) is 0.1 , and the pheromone bound in Eq. (14) lays in [0, 500]. Then place the ants at the originating node, put the current node to the tabu table and choose the next node according to the transition 
probability in Eq. (9) until the target is reached. Repeat this process until each ant in one generation completes the search. Once fixed number of iteration is reached, we update pheromone of the global best, otherwise update pheromone of the iteration best according to Eqs. (11) (13). Then limit the pheromone of Eq. (14), update the pheromone of edges and repeat the processes until the two algorithms were respectively executed for 150 iterations with the same parameters.

For comparing these two algorithms, we generate randomly the threat points and utilize the function voronoi() in Matlab to construct the environment of the battlefield, the results are displayed in Fig. 9.

From Fig. 9, we can see that, under the same circumstance, IACO in red only requires 37 iterations to converge, while ACO in blue takes 70 iterations, and therefore the former achieves $47 \%$ faster convergence rate and shows better performance on solving the problem of local optimum and low convergence rate. The two lines coincide at 70th iteration and stay stable after that, implying that the shortest paths found will not change. We also compare shortest weighted path length, convergence iteration and average weighted path length in Tab. 1. It indicates that IACO not only achieves higher convergence rate, but holds the same shortest weighted path length and lower average weighted path length than ACO. By IACO search algorithm, the average weighted path is close to the shortest weighted path showing the effectiveness and efficiency of the IACO.

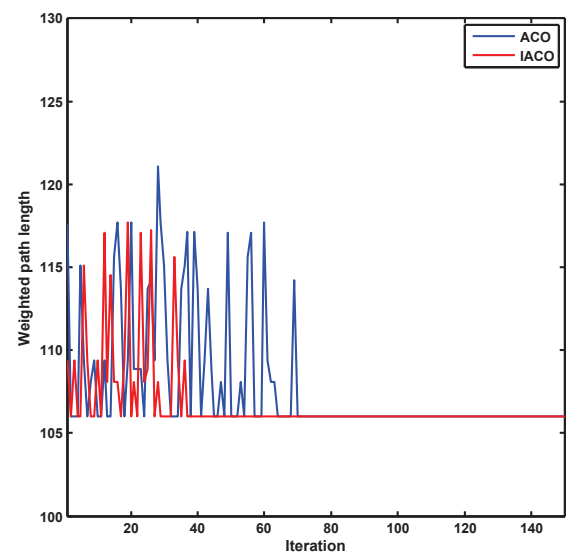

Figure 9: Comparison of ACO and IACO in weighted path length and iterations: ACO converges at 70th iteration and obtains shortest weighted path length, while IACO only takes 37 iterations to reach the same result.

Table 1: Comparison of ACO and IACO.

\begin{tabular}{|c|c|c|c|}
\hline $\begin{array}{c}\text { Path planning } \\
\text { method }\end{array}$ & $\begin{array}{c}\text { Shortest weighted } \\
\text { path length }\end{array}$ & $\begin{array}{c}\text { Average weighted } \\
\text { path length }\end{array}$ & $\begin{array}{c}\text { Convergence } \\
\text { iteration }\end{array}$ \\
\hline ACO & 106.02 & 108.06 & 70 \\
\hline IACO & 106.02 & 106.62 & 37 \\
\hline
\end{tabular}

\subsection{Smoothing the Initial Trajectory}

Coordination through smoothing is simulated in this subsection. Before the simulation is carried out, we assume only one
UAV exists in the battlefield flying from the initial location to the destination, and the angles of the initial trajectory requires to be smoothed to meet the UAV's turn constraint.

In this simulation, an initial path is supposed to be generated by the IACO, our proposed $k$-degree smoothing method and coordination strategy are applied to obtain the final and flyable path. The simulation results of the smoothed path using the $k$-degree smoothing method are shown in Fig. 10. The coordinates of the vertices of the plot in the bottom of Fig. 10 are $(10,10),(26,14),(28,34),(32,20),(44,22),(56,10),(70,22)$, $(72,6),(76,30),(84,32),(88,22)$, respectively. The other two plots are transformations of the bottom one in the vertical direction.

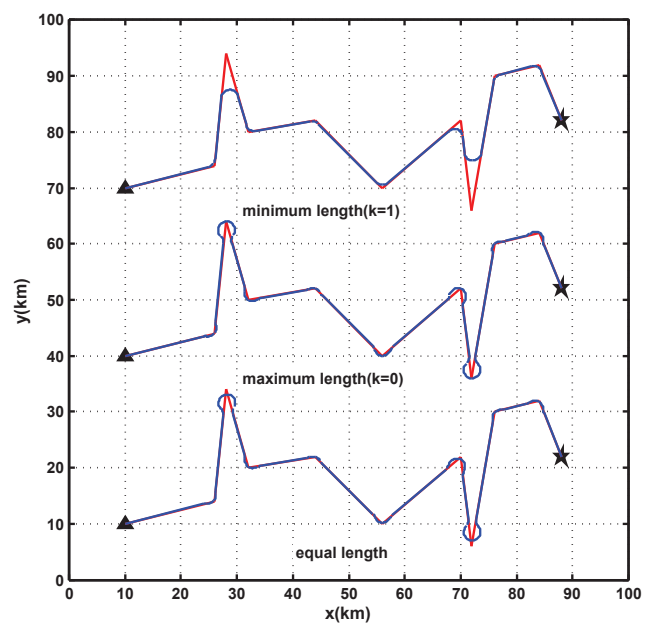

Figure 10: Smoothed path for single UAV: The red plot refers to the initial path, and the blue one refers to the smoothed path. Three plots respectively denotes three different smoothing strategies when the smoothed path is minimal, maximal and equal to the original path.

According to the previous section, the smoothed path length can be any value from the lower bound $(k=1)$ through the upper bound $(k=0)$, and the final smoothed path length is related only to the values of $k$ in each turn of the initial path. We have proven that given the difference between the desired qualified path length and the initial path length, the corresponding smoothing degree $k$ of each turn can be computed through a certain equation.

In the simulation, we first investigate the limit values of the smoothing degree $k$, shown as the upper two plots in Fig. 10. The initial path length is 158.20 , and we figure out the fact that when $k=1$, the UAV flies along the inscribed circle of the turning angle, thus making the smoothed path length minimal, which is 125.90 . Correspondingly, when $k=0$, the inscribed circle moves to the waypoints, which makes the smoothed path length maximal, which is 164.74. Therefore, the smoothed path length varies in the interval [125.90,164.74], and for a special case, the smoothed path has the same path length as that of the initial path.

By the theoretical result, we know that the three arcs of each angle guarantee the equal length between the smoothed path and the initial path. Additionally, as shown in the lowest plot of Fig.10, the increments or decrements in the path length 
contributed by each turn are all zero when the special case occurs, then we can easily calculate the corresponding values of $k$ by Eq. (27) in Theorem 1 of subsection 3.2, which are 0.391, 0.142, 0.372, 0.409, 0.368, 0.282, 0.114, 0.396, 0.374, respectively. In practical situations, the ultimate flyable path length distributes in the interval from the minimal to maximal. Since the smoothing process is completed, the simulations of multi-UAVs coordination can be operated.

\subsection{Simulation of Multi-UAVs Coordination}

In this subsection, multi-UAVs located at different start points are simulated to reach the target. In the following simulations, we randomly generate 16 nodes to represent the threats considered, then we carry out two simulations on the strong and weak coordination, respectively.

\subsubsection{Strong Coordination}

An air combat formation and two UAVs located at different start points are assigned to reach the same target simultaneously. Trajectories optimized in this simulation are presented in Fig. 11. Clearly, we can successfully plan paths for different UAVs to reach the same target from different start points simultaneously, and detailed information is given in Tab. 2. As displayed in Tab. 2, once the trajectory intervals for two UAVs are calculated, we can obtain their intersection [97.17,98.45], indicating that to achieve strong coordination, the ultimate path length of UAVs must be restricted within this range, and for expressing clearly, we take the intermediate value 97.82. Additionally, we can also see that ultimate paths for the two UAVs can be planned when the trajectory intersection is not empty and through smoothing the two UAVs could achieve strong coordination.

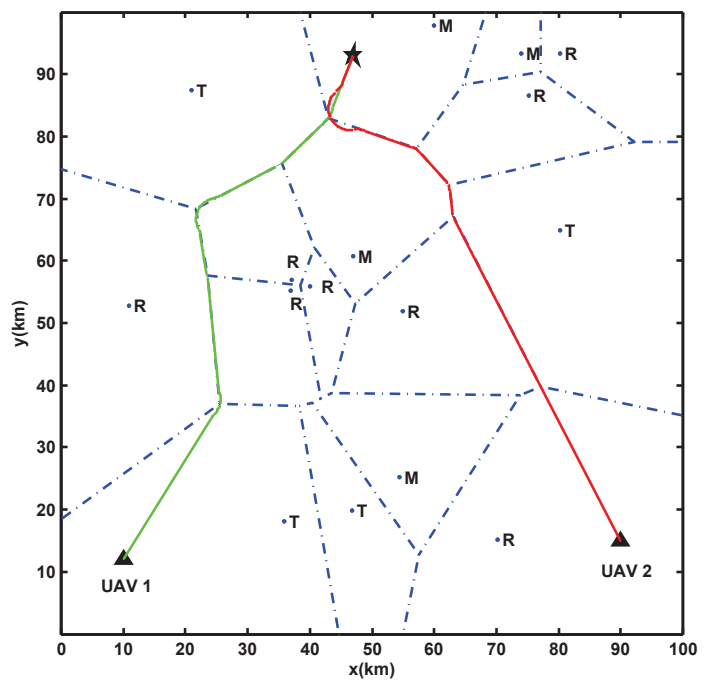

Figure 11: Strong coordination for two UAVs reaching the identical target: The triangle stands for the initial location and the star for destination, the green line denotes the final path for UAV1 and the red one for UAV2.

In another situation of strong coordination, two UAVs are assigned to reach different targets for mission demand from different start points. Trajectories optimized in this simulation are presented in Fig.12, we can successfully plan paths for two UAVs to reach different targets from different initial points simultaneously, and detailed information of the trajectories is given in Tab. 3. From Tab. 3, we can obtain the intersection of two UAVs [98.03,99.33], and according to the calculated trajectory intervals and its intermediate value 98.68, the strong coordination is obviously achieved.

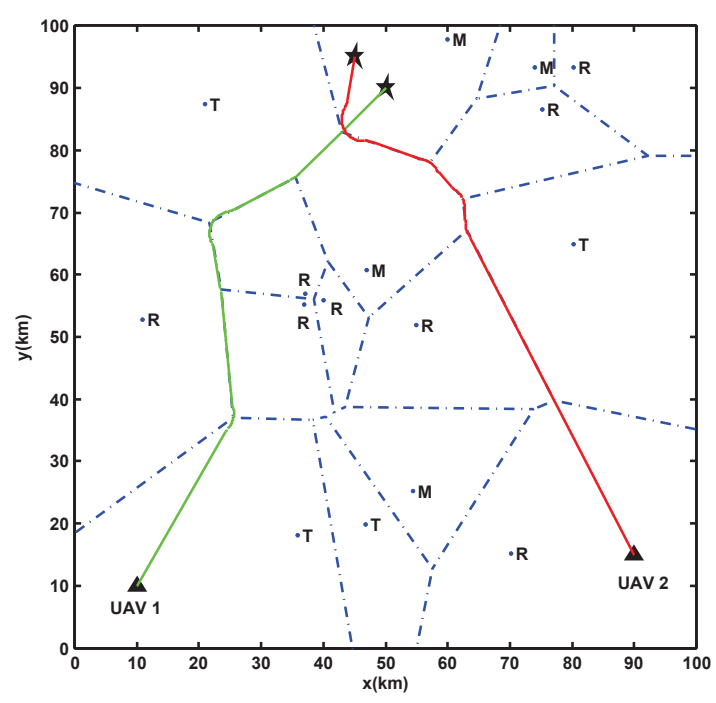

Figure 12: Strong coordination for two UAVs reaching different targets: The triangle stands for the initial location and the star for destination, the green line denotes the final path for UAV1 and the red one for UAV2.

In addition, an air combat formation composed of three UAVs and located at different sites is assigned to reach the same target simultaneously. Trajectories optimized in this simulation are presented in Fig. 13, and detailed information of the trajectories is given in Tab. 4. The intersection is $[60.67,60.72]$ and its intermediate value 60.70 is considered as the ultimate path length for each UAV to achieve the strong coordination.

The results above illustrate that the proposed strong coordination strategy can effectively solve multi-UAVs coordinated trajectory planning problems and allow multi-UAVs to arrive at the target location at the same time, i.e., multiple UAVs can complete tasks simultaneously. However, there are a lot of circumstances multi-UAVs cannot achieve strong coordination. Actually, that multi-UAVs reach the target location in a time interval could also be acceptable for some less urgent tasks, the corresponding simulations are shown in the following.

\subsubsection{Weak Coordination}

Two UAVs are assigned to reach the same target from different start points. While calculating the trajectory interval, we obtain that the trajectory interval for UAV1 is [89.47,90.74], and the trajectory interval for UAV2 is [91.23,91.43]. The intersection of the two intervals is empty implying that UAV1 and UAV2 cannot achieve strong coordination. Suppose the tolerate changes on path length $\Delta L(\Delta L=V \Delta t)$ is $2 \mathrm{~km}$, according to coordination strategy, weak coordination can be accomplished. Trajectories optimized in this simulation are presented in Fig.14, and detailed information of the trajectories is given 
Table 2: Information of strong coordination for two UAVs reaching the same target.

\begin{tabular}{|l|c|c|c|c|c|c|}
\hline & Starting point $(\mathrm{km})$ & Target $(\mathrm{km})$ & Initial path length $(\mathrm{km})$ & Trajectory interval $(\mathrm{km})$ & Intersection $(\mathrm{km})$ & Ultimate path length $(\mathrm{km})$ \\
\hline UAV 1 & $(10,12)$ & $(47,93)$ & 97.97 & {$[97.17,98.54]$} & \multirow{2}{*}{$907.17,98.45]$} & \\
\hline UAV 2 & $(90,15)$ & $(47,93)$ & 97.36 & {$[95.62,98.45]$} & \\
\hline
\end{tabular}

Table 3: Detailed information of strong coordination for two UAVs reaching different targets.

\begin{tabular}{|l|c|c|c|c|c|c|}
\hline & Starting point $(\mathrm{km})$ & Target $(\mathrm{km})$ & Initial path length $(\mathrm{km})$ & Trajectory interval $(\mathrm{km})$ & Intersection $(\mathrm{km})$ & Ultimate path length $(\mathrm{km})$ \\
\hline UAV 1 & $(10,10)$ & $(50,90)$ & 98.79 & {$[97.67,99.52]$} & \multirow{2}{*}[98.03,99.33]{} & \\
\hline UAV 2 & $(90,15)$ & $(45,95)$ & 98.77 & {$[98.03,99.33]$} & & \\
\hline
\end{tabular}

Table 4: Detailed information of strong coordination for three UAVs reaching the same target.

\begin{tabular}{|l|c|c|c|c|c|c|}
\hline & Starting point $(\mathrm{km})$ & Target $(\mathrm{km})$ & Initial path length $(\mathrm{km})$ & Trajectory interval $(\mathrm{km})$ & Intersection $(\mathrm{km})$ & Ultimate path length $(\mathrm{km})$ \\
\hline UAV 1 & $(18,21)$ & $(60,60)$ & 60.53 & {$[60.19,60.80]$} & \\
UAV 2 & $(77,3.5)$ & $(60,60)$ & 60.70 & {$[60.67,60.72]$} & \\
\hline UAV 3 & $(18,80)$ & $(60,60)$ & 60.83 & {$[60.67,60.72]$} & \\
6
\end{tabular}

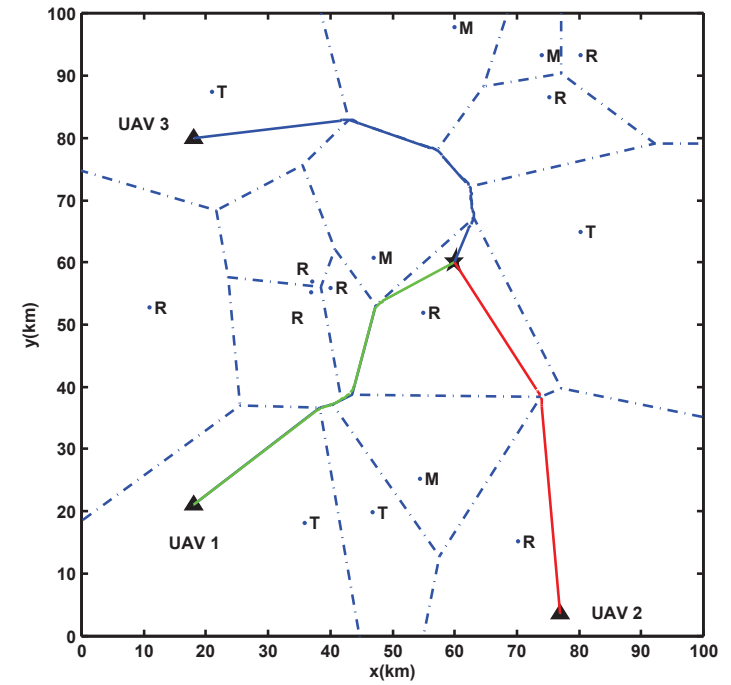

Figure 13: Strong coordination for three UAVs reaching the identical target: The triangle stands for the initial location and the star for destination, the green line denotes the final path for UAV1, the red one for UAV2 and the blue one for UAV3.

in Tab. 5. The ultimate path lengths for two UAVs are 90.74 and 91.23 respectively.

In the other situation of weak coordination, an air combat formation, consisting of three UAVs located at different sites, is set to reach the identical destination in an acceptable time interval. Through calculation, corresponding trajectory interval$\mathrm{s}$ for the three vehicles are [60.19,60.80], [61.19,61.22], and [60.68, 60.95], respectively. The hypothetical tolerate change on path length is $2 \mathrm{~km}$. Trajectories optimized in this simulation are presented in Fig. 15, and detailed information of the trajectories is given in Tab.6. The ultimate path lengths for three UAVs are $60.19,61.20,60.95$, respectively.

The above simulation results illustrate that the proposed weak coordination strategy can supplement strong coordination and help to solve the problem that multi-UAVs cannot reach

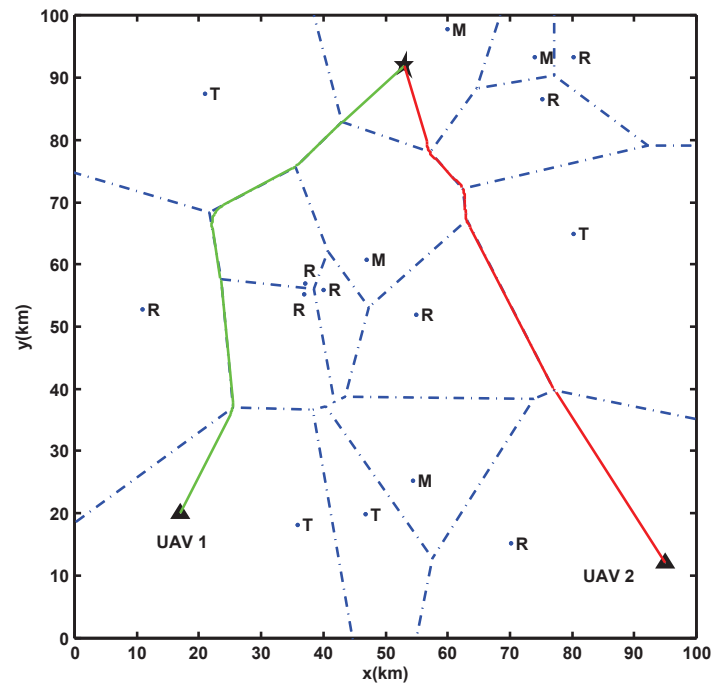

Figure 14: Weak coordination for two UAVs reaching the same target: The triangle stands for the initial location and the star for destination, the green line denotes the final path for UAV1 and the red one for UAV2.

targets simultaneously but in an acceptable time interval.

\section{Conclusions and Future Work}

In this paper, we propose a novel multi-UAVs coordinated path planning method based on $k$-degree smoothing. Based on Voronoi diagram, the proposed approach focuses on the redefinition of each edge cost. The UAV's initial path is obtained by Improved Ant Colony Optimization algorithm, and $k$-degree smoothing method is induced to make the planning path more flyable which leads to the coordination of multiple UAVs. Coordination between UAVs includes strong and weak conditions, depending on whether all agents arriving at the target location simultaneously or in an acceptable time interval. Simulation results validate the feasibility and effectiveness of the presented algorithm. 
Table 5: Detailed information of weak coordination for two UAVs reaching the same target.

\begin{tabular}{|c|c|c|c|c|c|c|}
\hline & Starting point $(\mathrm{km})$ & Target $(\mathrm{km})$ & Initial path length $(\mathrm{km})$ & Trajectory interval $(\mathrm{km})$ & Ultimate path length $(\mathrm{km})$ & Allowable error $(\mathrm{km})$ \\
\hline UAV 1 & $(17,20)$ & $(53,92)$ & 90.21 & {$[89.47,90.74]$} & 90.74 & \multirow{2}{*}{2} \\
\hline UAV 2 & $(95,12)$ & $(53,92)$ & 91.34 & {$[91.23,91.43]$} & 91.23 & \\
\hline
\end{tabular}

Table 6: Detailed information of weak coordination for three UAVs reaching the same target.

\begin{tabular}{|c|c|c|c|c|c|c|}
\hline & Starting point $(\mathrm{km})$ & Target $(\mathrm{km})$ & Initial path length $(\mathrm{km})$ & Trajectory interval (km) & Ultimate path length $(\mathrm{km})$ & Allowable error $(\mathrm{km})$ \\
\hline UAV 1 & $(18,21)$ & $(60,60)$ & 60.53 & {$[60.19,60.80]$} & 60.19 & \multirow{3}{*}{2} \\
\hline UAV 2 & $(77,3)$ & $(60,60)$ & 61.20 & {$[61.16,61.22]$} & 61.20 & \\
\hline UAV 3 & $(18,80)$ & $(60,60)$ & 60.83 & {$[60.68,60.95]$} & 60.95 & \\
\hline
\end{tabular}

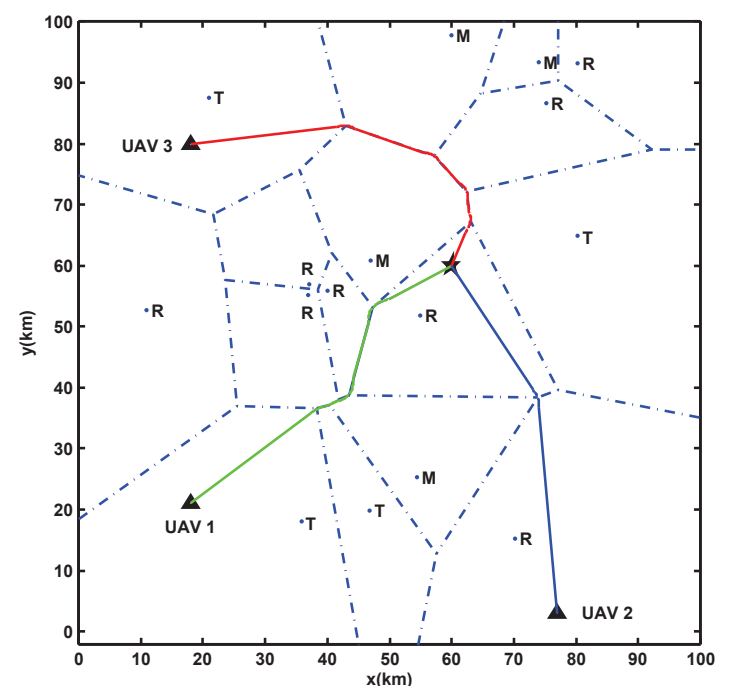

Figure 15: Weak coordination for three UAVs reaching the same target: The triangle stands for the initial location and the star for destination, the green line denotes the final path for UAV1, the blue one for UAV2 and the red one for UAV3.

However, our model has limitations in environment constructing, and therein we will focus on multi-UAVs coordinated path planning in complicated dynamic and uncertain situations for future research.

\section{Acknowledgements}

This work was supported in part by the National Science Foundation of China under Grants 61273308 and 61573081, and the China Scholarship Council.

\section{Reference}

[1] T. Shima, S.J. Rasmussen, A.G. Sparks, UAV cooperative multiple task assignments using genetic algorithms, in: American Control Conference, Jun 2005, pp. 2989-2994.

[2] J. Tisdale, Z. Kim, J.K. Hedrick, Autonomous UAV path planning and estimation, IEEE Robotics \& Automation Magzine, 61(2)(2009)35-42.

[3] V. Roberge, M. Tarbouchi, G. Labont, Comparison of parallel genetic algorithm and particle swarm optimization for real-time UAV path planning, IEEE Transactions on Industrial Informatics, 9(1)(2013)132-141.
[4] S. Ragi, E.K.P. Chong, UAV path planning in a dynamic environment via partially observable Markov decision process, IEEE Transactions on Aerospace and Electronic Systems, 49(4)(2013)2397-2412.

[5] X.Y. Zhang, H.B. Duan, An improved constrained differential evolution algorithm for unmanned aerial vehicle global route planning. Applied Soft Computing, 26(2015)270-284.

[6] M.A. Contreras-Cruz, V. Ayala-Ramirez, U.H. Hernandez-Belmonte, Mobile robot path planning using artificial bee colony and evolutionary programming, Applied Soft Computing, 30(2015)319-328.

[7] S. Ghosh, A. Halder, M. Sinha, Micro air vehicle path planning in fuzzy quadtree framework, Applied Soft Computing, 11(2011)4859-4865.

[8] N. Mathew, S.L. Smith, S.L. Waslander, Planning Paths for Package Delivery in Heterogeneous Multirobot Teams, IEEE Transactions on Automation Science and Engineering, 12(4)(2015)1298-1308.

[9] H. Qu, S.X. Yang, A.R. Willms, Y. Zhang, Real-Time Robot Path Planning Based on a Modified Pulse-Coupled Neural Networks Model, IEEE Transactions on Neural Networks, 20(11)(2009)1724-1739.

[10] R.E. Sehiemy, A.A. EI-Ela, A.A. Shaheen, Multi-objective fuzzy-based procedure for enhancing reactive power management, IET Generation, Transmission \& Distribution, 7(12)(2013)1453-1460.

[11] R.E. Precup, R.C. David, E.M. Petriu, et. al., Fuzzy logic-based adaptive gravitational search algorithm for optimal tuning of fuzzy-controlled servo systems, IET Control Theory \& Applications, 7(1)(2013)99-107.

[12] M.J. Gacto, M. Galende, R. Alcalá, F. Herrera, METSK-HD ${ }^{e}$ : A multiobjective evolutionary algorithm to learn accurate TSK-fuzzy systems in high-dimensional and large-scale regression problems, Information Sciences, 276(2014)63-79.

[13] N. Tomin, A. Zhukov, D. Sidorov, et. al., Random forest based model for reventing large-scale emergencies in power systems, International Journal of Artificial Intelligence, 13(1)(2015)211-228.

[14] F. Gu, Y.Q. He, J.D. Han, Active Persistent Localizaiton of a ThreeDimensional Moving Target Under Set-Membership Uncertainty Decsription Through Cooperation of Multiple Mobile Robots, IEEE Transactions on Industrial Eletronics, 62(8)(2015)4958-4971.

[15] M. C̆áp, P. Novák, A. Kleiner, M. Selecký, Prioritized Planning Algorith$\mathrm{m}$ for Trajectory Coordination of Multiple Mobile Robots, IEEE Transactions on Automation Science and Engineering, 12(3)(2015)835-849.

[16] H. Qu, X. Ke, T. Alexander, An improved genetic algorithm with coevolutionary strategy for global path planning of multiple mobile robots, Neurocomputing, 120(2013)509-517.

[17] P.B. Sujit, R. Beard, Cooperative path planning for multiple UAVs exploring an unknown region, in: American Control Conference, Jul 2007, pp. 347-352.

[18] D.Q. Zhu, H. Huang, S.X. Yang, Dynamic Task Assignment and Path Planning of Multi-AUV System Based on an Improved Self-Organizing Map and Velocity Synthesis Method in Three-Dimensional Underwater Workspace, IEEE Transactions on Cybernetics, 43(2)(2013)504-514.

[19] H. Ergezer, K. Leblebicioğlu, 3D path planning for multiple UAVs for maximum information collection, Journal of Intelligent \& Robotic Systems, 73(1)(2014)737-762.

[20] G. Zhao, K.F. Xuan, R. Wenny, et al., Voronoi-Based Continous k Nearest Neighbor Search in Mobile Navigation, IEEE Transactions on Industrial Electronics, 58(6)(2011)2247-2257.

[21] L. Vachhani, A.D. Mahindrakar, K. Sridharan, Mobile Robot Navigation Through a Hardware-Efficient Implementation for Control-Law-Based 
Construction of Generalized Voronoi Diagram, IEEE Transactions on Mchatronics, 16(6)(2011)1083-1095.

[22] P. Bhattacharya, I.T. Corp, A.B. Calgary, M.L. Gavrilova, RoadmapBased Path Planning Using the Voronoi Diagram for a Clearance-Based Shortest Path, IEEE Robotics \& Automation Magzine, 15(2)(2008)58-66.

[23] T. Sttzle, H.H. Hoos, MAX-MIN ant system, Future generation computer systems, 16(8)(2000)889-914. 


\section{Environment information}

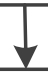

Construct Voronoi diagram $\&$ weight the edges

\section{UAV-1}

Initial Path planning with IACO $\downarrow$

K-degree smoothing

Path length interval

\begin{tabular}{|c|}
\hline UAV-1 \\
\hline $\begin{array}{c}\text { Initial Path planning } \\
\text { with IACO }\end{array}$ \\
\hline \multicolumn{1}{|}{$\mid$} \\
\hline K-degree smoothing \\
\hline Path length interval \\
\hline
\end{tabular}

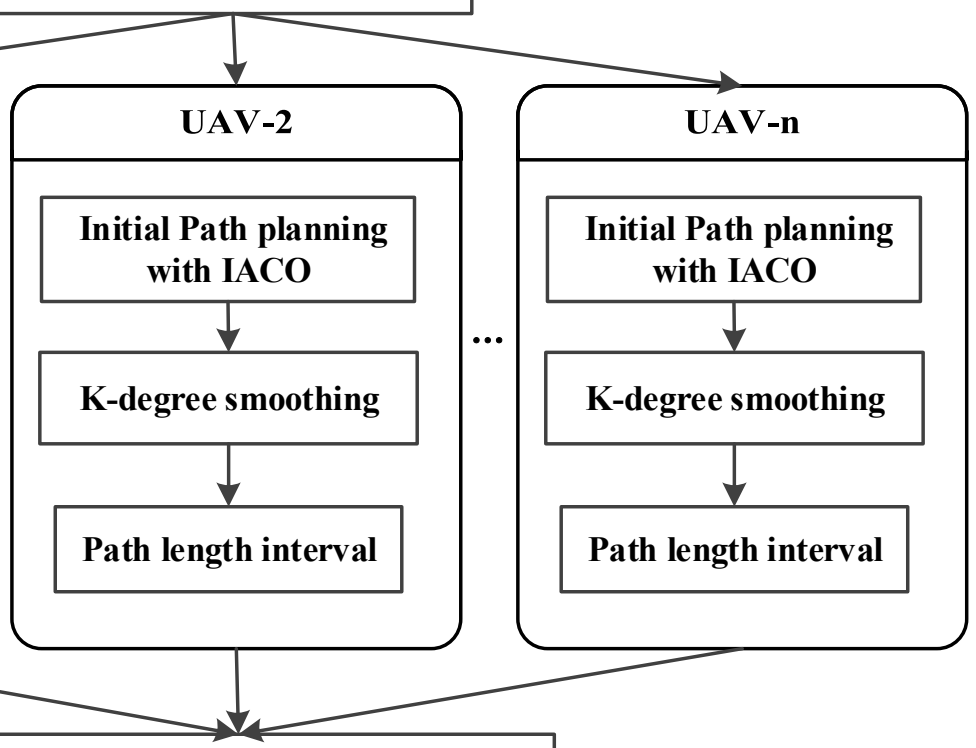

Operations on path length interval

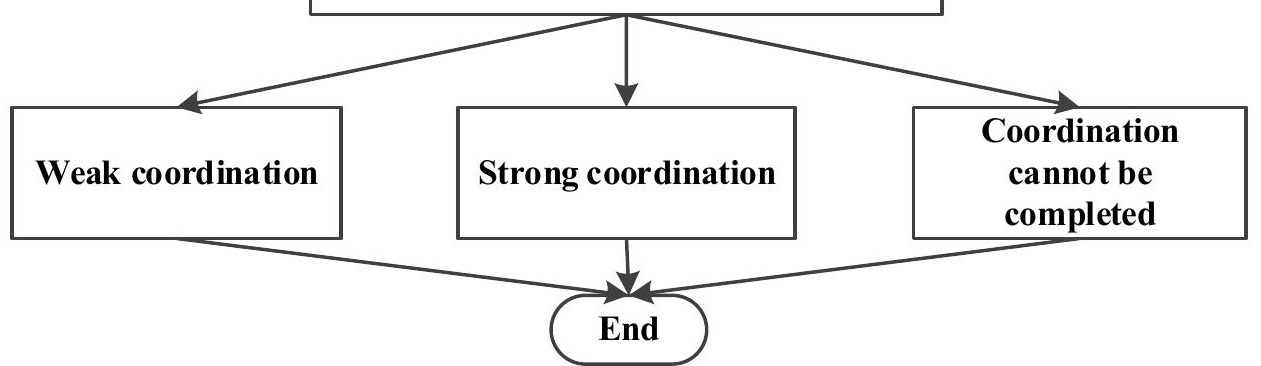

